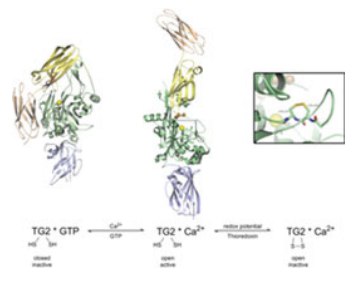
\title{
$1781 \begin{aligned} & \text { Regulation of the activities of the mammalian } \\ & \text { transglutaminase family of enzymes }\end{aligned}$ Cornelius Klöck and Chaitan Khosla
}

Mammalian transglutaminases catalyze post-translational modifications of their protein and peptide substrates at selected glutamine residues. As a group, the eight transglutaminases encoded by the human genome have diverse albeit poorly understood physiological and pathological roles. In each case, enzymatic activity is controlled on multiple levels by elaborate mechanisms. This review focuses on our emerging knowledge of the post-translational regulatory strategies, which range from cofactor binding to thiol-disulfide exchange and proteolytic activation. Of particular interest are the keratinocyte (TG1), tissue (TG2), and epidermal (TG3) transglutaminases, and Factor XIII.

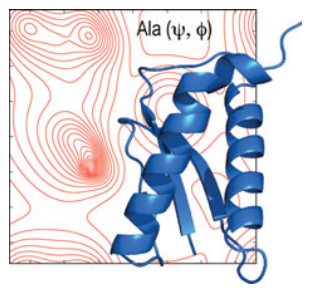

\section{Smooth statistical torsion angle potential derived from a large conformational database via adaptive kernel density estimation improves the quality of NMR protein structures Guillermo A. Bermejo, G. Marius Clore, and Charles D. Schwieters}

Databases of high-resolution X-ray protein models may be used to bias the calculation of new protein structures toward features frequently observed in the database, thus reducing the effect of incomplete experimental information. In this vein, the authors have developed a potential that biases backbone and side-chain torsion-angle combinations toward those present in a database of more than a million quality-filtered amino acid conformations. Their approach yields smooth yet fully featured energy surfaces that, when added to standard NMR structure calculation protocols, improve conformation, atomic interactions (clashes, packing), and structure accuracy. We show that the new potential clearly outperforms a widely used older implementation.

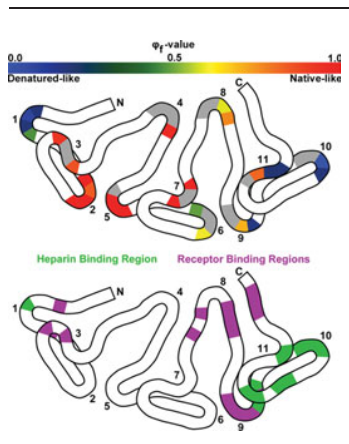

\section{Experimental support for the foldability-function tradeoff hypothesis: Segregation of the folding nucleus and functional regions in fibroblast growth factor-1 Liam Longo, Jihun Lee, and Michael Blaber}

The acquisition of protein function has long been associated with accommodation at the expense of thermodynamic stability. In this article, Longo et al. show that the price of function is steeper than originally thought: not only are functional residues destabilizing but also they are associated with reduced foldability. This study marks the first experimental observation of a "foldability-function tradeoff" by $\varphi$-value analysis and reveals a potential evolutionary advantage of symmetric protein folds, in which structural symmetry may enable folding pathway redundancy.

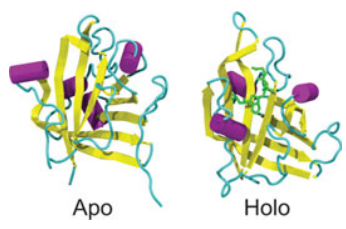

\section{The binding mechanism, multiple binding modes, and allosteric regulation of Staphylococcus aureus Sortase A probed by molecular dynamics simulations Kalli Kappel, Jeff Wereszczynski, Robert T. Clubb, and J. Andrew McCammon}

Sortase enzymes, which anchor extracellular proteins into the cell wall of Gram-positive bacteria, function by recognizing a conserved sorting sequence in their target protein, cleaving its backbone at this site, and catalyzing its linkage to a lipid II molecule. In this article, the authors have used conventional and accelerated molecular dynamics simulations to examine the process by which a sortase protein interacts with its pentapeptide substrate. The results show that there are multiple stable binding poses for the substrate reveal allosteric networks throughout the protein and shed new light on the recognition mechanism of this emerging antibacterial target. 